\title{
Internacionalidad de las revistas de psicología multidisciplinar editadas en iberoamérica e incluidas en la Web of Science*
}

\author{
Internationality of the Multidisciplinary Psychology Journals \\ Published in Ibero-America and Included in the Web Of Science

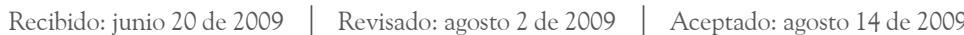 \\ IZABELA ZYCH ${ }^{* *}$ \\ GUALBERTO BUELA-CASAL *** \\ Universidad de Granada, Granada, España
}

" Artículo de investigación en cienciometría.

** Dirección de contacto: Campus Cartuja s/n, 18011 Granada, España.

Correo electrónico: izabela@ugr.es

**** Campus Cartuja s/n, 18011, Granada, España. Correo electrónico: gbuela@ugr.es

\section{RES UMEN}

Las revistas internacionales se consideran más prestigiosas y de mayor calidad que las nacionales, aunque su significado no se había establecido hasta hace poco. Es más, el criterio de internacionalidad se utiliza para tomar importantes decisiones como por ejemplo en la evaluación del personal docente, universidades, programas de doctorado etc., aún sin conocer su significado exacto. Por tanto, el presente estudio utiliza los criterios de internacionalidad establecidos en una encuesta a más de 16.000 científicos de todo el mundo, para la elaboración de un Índice de Internacionalidad de las revistas de psicología multidisciplinar incluidas en la Web of Science. Los resultados demuestran que la revista con mayor nivel de internacionalidad es la Revista Latinoamericana de Psicología, seguida por The Spanish Journal of Psychology, Psicothema y Universitas Psychologica. Todas las revistas cumplen con los criterios de internacionalidad y el estudio proporciona resultados de gran relevancia para potenciarla.

Palabras clave autores

Internacionalidad, revistas científicas, revistas de psicología.

Palabras clave descriptor

Revistas científicas, revistas de psicología.

\section{A B S T R A C T}

International journals are considered more prestigious and with more quality than national ones, although the meaning of this had not been established until recently. Moreover, internationality has been used as a criterion to make important decisions, for example to assess the performance of scientists, universities, doctoral degree programs, and so on, without knowing the exact meaning of internationality. Thus, the current study uses the criteria of internationality established by a survey on more than 16,000 scientists from all over the world to create an Internationality Index of multidisciplinary psychology journals published in Ibero-America and included in the Web of Science. Results show that the most international journal is Revista Latinoamericana de Psicología, followed by The Spanish Journal of Psychology, Psicothema and Universitas Psychologica. All the journals fulfill the criteria for internationality and the study provides very important results which will allow its increase.

Key words authors

Internationality, Scientific Journals, Psychology Journals.

Key words plus

Science-Periodicals, Psychology Journals. 


\section{Introducción}

Desde hace décadas, las revistas internacionales son más prestigiosas y más valoradas que las nacionales. No obstante, hasta hace poco no se había establecido el significado de este término. No se trata de una simple palabra dado que es un concepto de gran importancia que aparece, por ejemplo, en los criterios para la selección del personal docente y evaluación de su productividad (BuelaCasal \& Sierra, 2007; Musi-Lechuga, Olivas-Ávila, Portillo-Reyes \& Villalobos-Galvis, 2005; Sierra, Buela-Casal, Bermúdez \& Santos, 2008, 2009a, 2009b; Viñolas, Aguado, Josa, Villegas \& Fernández Prada, 2009), en los estudios de evaluación de los centros de investigación (Buela-Casal, 2005; Buela-Casal \& Castro, 2009; Buela-Casal et al., 2009; Del Río Bermúdez, 2008; Muñiz \& Fonseca-Pedrero, 2008; Zárraga, Balluerka \& Ferreira, 2009), en la distinción entre publicaciones internacionales y bases de datos internacionales (Fernández-Rios \& Buela-Casal, 2009); en los rankings de las universidades (Buela-Casal, Bermúdez, Sierra, Quevedo \& Castro, 2009; Buela-Casal, Gutiérrez, Bermúdez \& Vadillo, 2007; Pagani et al., 2006) o en los criterios de evaluación de los programas de doctorado (Bengoetxea \& Arteaga, 2009; Buela-Casal \& Castro, 2008a, 2008b; Castro \& Buela-Casal, 2008; Gil Roales-Nieto, 2009; Musi-Lechuga, Olivas-Ávila \& Buela-Casal, 2009), o con la docencia (García-Berro, Dapia, Amblàs, Bugeda \& Roca, 2009; González Jiménez, Macías Gómez, Rodríguez Sánchez \& Aguilera García, 2009), relacionado con la financiación (Carrillo de Albornoz, 2009; Rodríguez-Marín et al., 2009) o incluso se podría relacionar con los índices de cocitación entre las revistas (García-Martínez, Guerrero-Bote, Hassan-Montero \& Moya-Anegón, 2009) o sobre los criterios de selección de las revistas para su inclusión en la Web of Science (Garfield, 2003; Ruiz-Pérez, Delgado \& JiménezContreras, 2006), o comparando la proyección nacional e internacional de las publicaciones y las tendencias del factor de impacto (Delgado, Jiménez \& Ruiz, 2009; Jiménez-Contreras, Delgado,
Ruiz, Rodríguez-García \& Moneda, 2009; OscaLluch et al., 2005).

Aunque el término apareciese en todos estos criterios, cada evaluador lo entendía a su manera, ya que hasta hace poco no existía una forma objetiva de medirlo. Hay quien entendía que una revista internacional era la que se publicaba fuera del país; sin embargo, no es lo mismo una revista extranjera que una internacional y en una misma revista puede haber autores nacionales y extranjeros, por ejemplo, Aceros Gualdrón y Evnitskaya (2008) de España; Farkas-Klein (2008) de Chile; Fernández Arata (2008) de Perú; Herrera y Guarino (2008) de Venezuela; Ison-Zintilini y MorelatoGiménez (2008) de Argentina; Laranjeira (2008) de Portugal; Ruiz Carrillo y Estrével Rivera (2008) de México; Teodoro, Cerqueira-Santos, Araujo de Morais y Koller (2008) de Brasil; Valenzuela (2009) de Bélgica o Annicchiarico, Gutiérrez, Ortega y Barragán (2009) de los Estados Unidos, en el caso de la revista Universitas Psychologica. Otro criterio utilizado con mucha frecuencia es la inclusión de la revista en la Web of Science [WOS], la base de datos de revistas más prestigiosa en el mundo. No obstante, se trata de una base de datos que mide el Factor de Impacto de las revistas que está basado en el número de citas. Es evidente que una revista internacional, aparte de estar incluida en la WOS tiene que cumplir con más criterios (Buela-Casal, 2001; Buela-Casal, Perakakis, Taylor \& Checa, 2006).

Por todas las razones mencionadas anteriormente, Zych y Buela-Casal (2009) establecieron los criterios de internacionalidad, mediante una encuesta respondida por más de 16.000 científicos procedentes de 109 países y 9 campos de conocimiento de la UNESCO. Por primera vez, los científicos, directores de las revistas y los evaluadores podrán conocer cuál es el nivel la internacionalidad de cada publicación, utilizando los criterios procedentes de un estudio con una muestra lo suficientemente amplia como para asegurarse de que éstos son objetivos y que representan la opinión de la comunidad científica mundial. Estos mismos autores propusieron cómo calcular el Índice de In- 
ternacionalidad siguiendo estos criterios (Zych \& Buela-Casal, 2009). Se han calculado sus valores para las revistas españolas de psicología (BuelaCasal, Zych, Sierra \& Bermúdez, 2007) y para las revistas iberoamericanas de psicología incluidas en la WOS (Zych \& Buela-Casal, 2007), cuando sólo eran tres. Hoy en día, ya son nueve las revistas incluidas sólo en el campo de psicología multidisciplinar: Anales de Psicología, Estudios de Psicología, Psicologia-Reflexao e Critica, Psicothema, Revista Latinoamericana de Psicología Fundamental, Revista Latinoamericana de Psicología, Revista Mexicana de Psicología, Spanish Journal of Psychology y Universitas Psychologica. Teniendo en cuenta que todas estas revistas han entrado a formar parte de esta prestigiosa base de datos, es importante llevar a cabo un análisis de la internacionalidad de las mismas. Por tanto, el presente trabajo es la respuesta a esta necesidad dado que consiste en calcular el Índice de Internacionalidad de las revistas de psicología multidisciplinar editadas en Iberoamérica e incluidas en la WOS.

\section{Método}

\section{Unidad de Análisis}

Se analizaron las revistas iberoamericanas de psicología multidisciplinar editadas en Iberoamérica e incluidas en la Web of Science. En total, se analizaron nueve revistas:

- Anales de Psicología (España)

- Estudios de Psicología (España)

- Psicologia-Reflexao e Critica (Brasil)

- Psicothema (España)

- Revista Latinoamericana de Psicología (Colombia)

- Revista Latinoamericana de Psicopatología Fundamental (Brasil)

- Revista Mexicana de Psicología (México)

- The Spanish Journal of Psychology (España)

- Universitas Psychologica (Colombia)
Los siguientes criterios de internacionalidad establecidos por Buela-Casal y Zych (2009) fueron utilizados en el estudio:

- El idioma de publicación

- El Factor de Impacto ISI

- Los países de procedencia de los miembros del comité editorial

- La inclusión en JCR

- La procedencia de los autores

- Las normas de publicación

- Acceso por Internet

- Acceso por Internet gratuito

- Inclusión en las bases de datos

- La pertenencia de la revista a asociaciones con la palabra "internacional" en los nombres

- La inclusión de la palabra "internacional" en el nombre de la revista

\section{Materiales}

- Revistas Iberoamericanas de Psicología multidisciplinar incluidas en la Web of Science

- La base de datos Thomson (ISI)

\section{Diseño y procedimiento}

El presente artículo es un estudio descriptivo mediante análisis de documentos (Montero \& León, 2007) redactado según las normas establecidas por Ramos-Álvarez, Moreno-Fernández, ValdésConroy y Catena (2008) y los Principios de Berlín para Rankings de Instituciones de Educación Superior (Internacional Ranking Expert Group, 2006).

Basándose en los criterios de la internacionalidad establecidos por Buela-Casal y Zych (2009) y la forma de calcular el Índice de Internacionalidad propuesta por estos mismos autores (Zych \& BuelaCasal, 2009) se han analizado las revistas iberoamericanas de psicología multidisciplinar incluidas en la WOS. Los años incluidos en el análisis de los once criterios mencionados anteriormente fueron el 2007 y el 2008. Primero se buscó la información mediante Internet y si ésta no se encontraba, se analizaron las versiones en papel. Finalmente, también se contactó con los directores de las revistas 
para pedir que proporcionaran los datos, en caso de no encontrarlos. Se creó una base de datos en la que se incluyeron todos los criterios y subcriterios cumplidos por cada revista. Se calcularon las puntuaciones en cada uno de éstos y se ordenaron las revistas según sus puntuaciones totales en el Índice de Internacionalidad.

\section{Resultados}

El Índice de Internacionalidad de las revistas de psicología multidisciplinar editadas en Iberoamérica e incluidas en la Web of Science se presenta en la Tabla 1.

TABLA 1

Índice de Internacionalidad de las revistas de psicología multidisciplinar editadas en Iberoamérica e incluidas en la Web of Science

\begin{tabular}{|c|c|c|c|c|c|c|c|c|c|c|c|c|}
\hline $\begin{array}{l}\text { Nombre de } \\
\text { la revista }\end{array}$ & $\begin{array}{l}\text { Idioma } \\
\text { de } \\
\text { publi- } \\
\text { cación }\end{array}$ & $\begin{array}{c}\text { Ac- } \\
\text { ceso } \\
\text { inter- } \\
\text { net }\end{array}$ & $\begin{array}{l}\text { Nor- } \\
\text { mas de } \\
\text { publi- } \\
\text { cación }\end{array}$ & JCR & $\begin{array}{c}\text { Bases } \\
\text { de } \\
\text { datos }\end{array}$ & $\begin{array}{c}\text { Co- } \\
\text { mité } \\
\text { edito- } \\
\text { rial }\end{array}$ & $\begin{array}{c}\text { Acceso } \\
\text { por } \\
\text { internet } \\
\text { gratuito }\end{array}$ & $\begin{array}{c}\text { Factor } \\
\text { de Im- } \\
\text { pacto }\end{array}$ & $\begin{array}{c}\text { Proce- } \\
\text { dencia } \\
\text { de los } \\
\text { autores }\end{array}$ & $\begin{array}{c}\text { Organi- } \\
\text { zación } \\
\text { interna- } \\
\text { cional }\end{array}$ & $\begin{array}{l}\text { "Interna- } \\
\text { cional” } \\
\text { en el } \\
\text { nombre }\end{array}$ & Total \\
\hline $\begin{array}{l}\text { Revista } \\
\text { Latinoame- } \\
\text { ricana de } \\
\text { Psicología }\end{array}$ & 6,02 & 10,97 & 10,56 & 9,96 & 7,94 & 9,71 & 9,68 & 4,56 & 3,08 & 0 & 0 & 72,48 \\
\hline $\begin{array}{l}\text { The Spanish } \\
\text { Journal of } \\
\text { Psychology }\end{array}$ & 4,20 & 10,97 & 10,56 & 9,96 & 9,45 & 7,28 & 9,68 & 4,56 & 2,31 & 0 & 0 & 68,97 \\
\hline Psicothema & 6,00 & 10,97 & 10,56 & 9,96 & 6,80 & 7,28 & 9,68 & 6,85 & 0,77 & 0 & 0 & 68,87 \\
\hline $\begin{array}{l}\text { Universisi- } \\
\text { tas Psycho- } \\
\text { logica }\end{array}$ & 6,02 & 10,97 & 10,56 & 9,96 & 9,83 & 5,66 & 9,68 & $2,28 *$ & 1,54 & 0 & 0 & 66,50 \\
\hline $\begin{array}{l}\text { Anales de } \\
\text { Psicologia }\end{array}$ & 6,00 & 10,97 & 10,56 & 9,96 & 6,80 & 4,85 & 9,68 & $2,28^{*}$ & 2,31 & 0 & 0 & 63,41 \\
\hline $\begin{array}{l}\text { Revista } \\
\text { Latinoame- } \\
\text { ricana de } \\
\text { Psicopatolo- } \\
\text { gía Funda- } \\
\text { mental }\end{array}$ & 7,95 & 10,97 & 10,56 & 9,96 & 3,78 & 4,85 & 9,68 & $2,28 *$ & 0,77 & 0 & 0 & 60,80 \\
\hline $\begin{array}{l}\text { Estudios de } \\
\text { Psicología }\end{array}$ & 6,00 & 10,97 & 10,56 & 9,96 & 2,65 & 6,47 & 4,84 & $2,28 *$ & 2,31 & 0 & 0 & 56,04 \\
\hline $\begin{array}{l}\text { Psicologia- } \\
\text { Reflexao e } \\
\text { Critica }\end{array}$ & 0,02 & 10,97 & 10,56 & 9,96 & 4,54 & 5,66 & 9,68 & $2,28 *$ & 0,77 & 0 & 0 & 54,44 \\
\hline $\begin{array}{l}\text { Revista } \\
\text { Mexicana } \\
\text { de Psicolo- } \\
\text { gía }\end{array}$ & 1,80 & 5,48 & 10,56 & 9,96 & 3,02 & 6,47 & 4,84 & 2,28 & 3,08 & 0 & 0 & 47,49 \\
\hline
\end{tabular}

* Se han asignado valores equivalentes al cuarto cuartil dado que el Factor de Impacto todavía no se conoce.

Fuente: elaboración propia. 
Tal como se puede apreciar, la revista con mayor nivel de internacionalidad es Revista Latinoamericana de Psicología, seguida por The Spanish Journal of Psychology, Psicothema y Universitas Psychologica.

La revista cuyos artículos se editan en el mayor número de idiomas es la Revista Latinoamericana de Psicopatología Fundamental (portugués, inglés, francés y español). Las revistas analizadas tienen acceso a sus artículos por Internet, incluyendo el último número, a excepción de la Revista Mexicana de Psicología, con acceso a los resúmenes y no a artículos completos. Todas las revistas cumplen con las normas de publicación y están incluidas en los Journal Citation Reports, aunque el valor en éste no ha sido todavía calculado para Universitas Psychologica, Anales de Psicología, Revista Latinoamericana de Psicología Fundamental, Estudios de Psicología y Psicología-Reflexao e Critica. La revista incluida en el mayor número de bases de datos es la Universitas Psychologica (26 bases de datos) y el mayor número de países de procedencia de los miembros del comité editorial se encontró en la Revista Latinoamericana de Psicología (12 países). Estudios de Psicología y Revista Mexicana de Psicología tienen acceso gratuito por Internet a resúmenes, mientras que en caso de todas las restantes se puede acceder a artículos completos, incluyendo el último número. En cuanto al Factor de Impacto, como ya se ha mencionado, no es conocido para todas las revistas y se asignó valores correspondientes a cuarto cuartil en caso de las que todavía no lo tienen calculado. La revista con mayor puntuación en este criterio es Psicothema incluida en el segundo cuartil ${ }^{1}$ de WOS. Las revistas con mayor ratio de países de procedencia de los autores/número total de artículos son la Revista Latinoamericana de Psicología y la Revista Mexicana de Psicología (cerca de 0,2). No hay ninguna revista que contenga la palabra "internacional" en el nombre o en el nombre de la institución a la que pertenece. 1 Los cuartiles se entienden de tal forma que el primero es el de
mayor puntuación y el cuarto el de menor.

\section{Discusión}

El presente estudio es la primera clasificación de las revistas iberoamericanas incluidas en la categoría de psicología multidisciplinar en la Web of Science en cuanto a su internacionalidad. Gracias a ésta, tanto los autores como los directores de las mismas podrán conocer cuál es la situación, saber si la revista es o no internacional y además, tendrán la oportunidad de mejorar su nivel, conociendo exactamente sus criterios junto con los puntos fuertes y débiles mostrados en los resultados de este estudio. Como podemos ver en el ejemplo de un editorial de la revista Universitas Psychologica (López López, 2008), los directores necesitan trabajar día tras día para mejorar la revista. Gracias a este estudio, será más fácil conseguir este objetivo pues facilita información relevante para ello.

Tal como se describe en la introducción del presente estudio, el número de revistas iberoamericanas incluidas en la WOS ha crecido notablemente y hoy en día son ya dieciocho en total, de las que nueve están en la categoría multidisciplinar. En esta época de globalización y libre flujo de información, cada vez son más los países que participan en la producción científica mundial. Parece que la dominancia anglosajona está disminuyendo, lo cual se refleja en la presencia de estas nueve revistas iberoamericanas en la categoría de psicología multidisciplinar de la WOS, de las que sólo una publica artículos exclusivamente en inglés, siete admiten español y cuatro portugués. Hace relativamente pocos años, Ardila $(1982,2002)$ comentaba la dominancia de la psicología anglosajona y quizás esta situación está cambiando. En la misma línea, un reciente análisis cuantitativo, por países, de la productividad en psicología de las revistas incluidas en la WOS mostró un crecimiento de la producción científica en España, varios países latinoamericanos en vías de desarrollo y otros Estados no anglosajones; así, por ejemplo, la Psicología española ocupa el noveno puesto a nivel mundial y el quinto en la Unión Europea en artículos en la WOS (Navarrete-Cortés, Quevedo-Blasco, Chaichio-Moreno, Ríos \& Buela-Casal, 2009). Por ello, hoy en día es incluso más importante demostrar la 
internacionalidad de las revistas iberoamericanas, y el presente estudio añade un conocimiento importante en este ámbito.

\section{Referencias}

Aceros Gualdrón, J. C. \& Evnitskaya, N. (2008). La identidad colectiva en la interacción: análisis de un encuentro comunicativo entre activistas tecnológicos. Universitas Psychologica, 7(1), 57-71.

Annicchiarico, I., Gutiérrez, G., Ortega, L. A. \& Barragán, B. (2009). Medición indirecta de la liberación de andrógenos y su relación con la conducta sexual en coturnix japonica. Universitas Psychologica, 8(2), 497-506.

Ardila, R. (1982). International psychology. American Psychologist, 37, 323-329.

Ardila, R. (Ed.). (2002). La psicología en el futuro. Pirámide: Madrid.

Bengoetxea, E. \& Arteaga, J. (2009). La evaluación de posgrados internacionales en la Unión Europea. Ejemplos de buenas prácticas de programas europeos. Revista de Universidad y Sociedad del Conocimiento (RUSC), 6(2), 60-68.

Buela-Casal, G. (2001). La psicología española y su proyección internacional, el problema del criterio: internacional, calidad y castellano y/o inglés. Papeles del Psicólogo, 79, 53-57.

Buela-Casal, G. (2005). Situación actual de la productividad científica de las universidades españolas. International Journal of Clinical and Health Psychology, 5, 175-190.

Buela-Casal, G., Bermúdez, M. P., Sierra, J. C., Quevedo, R. \& Castro, A. (2009). Ranking de productividad en investigación de las universidades públicas españolas. Psicothema, 21, 309-317.

Buela-Casal, G. \& Castro, A. (2008a). Análisis de la evolución de los programas de doctorado con Mención de Calidad en las universidades españolas y pautas para su mejora. Revista de Investigación en Educación, 5, 49-60.

Buela-Casal, G. \& Castro, A. (2008b). Criterios y estándares para la obtención de la Mención de Calidad en Programas de Doctorado: evolución a través de las convocatorias. International Journal of Psychology and Psychological Therapy, 8, 127-136.

Buela-Casal, G. \& Castro, A. (2009). Las tecnologías de la información y la comunicación y la evaluación de la calidad en la educación superior. Revista de Universidad y Sociedad del Conocimiento (RUSC), $6(2), 3-8$.

Buela-Casal, G, Gutiérrez, O., Bermúdez, M. P. \& Vadillo, O. (2007). Comparative study of international academic rankings of universities. Scientometrics, 71, 349-365.

Buela-Casal, G., Perakakis, P., Taylor, M. \& Checa, P. (2006). Measuring internationality: Reflections and perspectives on academic journals. Scientometrics, 67, 45-65.

Buela-Casal, G. \& Sierra, J. C. (2007). Criterios, indicadores y estándares para la acreditación de profesores titulares y catedráticos de Universidad. Psicothema, 19, 537-551.

Buela-Casal, G., Vadillo, O., Pagani, R., Bermúdez, M. P., Sierra, J. C., Zych, I. et al. (2009). Comparación de los indicadores de la calidad de las universidades. Revista de Universidad y Sociedad del Conocimiento (RUSC), 6(2), 9-21.

Buela-Casal, G. \& Zych, I. (2009). The first reliable measure of the internationality of the scientific publications. En revisión.

Buela-Casal, G., Zych, I., Sierra, J. C. \& Bermúdez, M. P. (2007). The Internationality Index of the Spanish Psychology Journals. International Journal of Clinical and Health Psychology, 7, 899-910.

Castro, A. \& Buela-Casal, G. (2008). La movilidad de profesores y estudiantes en programas de postgrado: ranking de las universidades españolas. Revista de Investigación en Educación, 5, 61-74.

Del Río Bermúdez, L. (2008). Cómo implantar y certificar un sistema de gestión de la calidad en la Universidad. Revista de Investigación en Educación, 5, 5-11.

Delgado, E., Jiménez, E. \& Ruiz, R. (2009). La ciencia española a través de la Web of Science (1996. 2007). El Profesional de la Información, 18, 437-443.

Farkas-Klein, C. (2008). Escala de evaluación prenatal (EEP): desarrollo, propiedades psicométricas y aplicaciones. Universitas Psychologica, 7(2), 457467. 
Fernández Arata, J. M. (2008). Desempeño docente y su relación con la orientación a la meta, estrategias de aprendizaje y autoeficacia: un estudio con maestros de primaria de Lima, Perú. Universitas Psychologica, 7(2), 384-402.

Fernández-Rios, L. \& Buela-Casal, G. (2009). Standards for the preparation and writing of Psychology review articles. International Journal of Clinical and Health Psychology, 9, 329-344.

García-Berro, E., Dapia, F., Amblàs, G., Bugeda G. \& Roca, S. (2009). Estrategias e indicadores para la evaluación de la docencia en el marco del EEES. Revista de Investigación en Educación, 6, 142-152.

García-Martínez, A. T., Guerrero-Bote, V., HassanMontero, Y. \& Moya-Anegón, F. (2009). La Psicología en el dominio científico español a través del Análisis de Cocitación de Revistas. Universitas Psychologica, 8(1), 13-26.

Garfield, E. (2003). The meaning of the impact factor. International Journal of Clinical and Health Psychology, 3, 363-369.

Gil Roales-Nieto, J. (2009). Análisis de los estudios de doctorado en psicología con mención de calidad en universidades españolas. Revista de Investigación en Educación, 6, 160-172.

González Jiménez, F. E., Macías Gómez, E., Rodríguez Sánchez, M. \& Aguilera García, J. L. (2009). Prospectiva y evaluación del ejercicio docente de los profesores universitarios como exponente de buena calidad. Revista de Universidad y Sociedad del Conocimiento (RUSC), 6(2), 38-48.

Herrera, V. \& Guarino, L. (2008). Sensibilidad emocional, estrés y salud percibida en cadetes navales venezolanos. Universitas Psychologica, 7(1), 185-198.

Internacional Ranking Expert Group. (2006). Berlin Principles on Ranking of Higher Education Institutions. Disponible en http://www.che.de/downloads/ Berlin_Principles_IREG_534.pdf

Ison-Zintilini, M. S. \& Morelato-Giménez, G. S. (2008). Habilidades socio-cognitivas en niños con conductas disruptivas y víctimas de maltrato. Universitas Psychologica, 7(2), 357-367.

Jiménez-Contreras, E., Delgado, E., Ruiz, R., Rodríguez-García, G. \& Moneda, M. (2009). Spanish psychology journals: Demography, editorial ten- dencies and impact. Psychology Science Quarterly, 51, 100-118.

Laranjeira, C. A. (2008). Tradução e validação portuguesa do revised life orientation test (LOT-R). Universitas Psychologica, 7(2), 469-476.

López López, W. (2008). Editorial. Universitas Psychologica, 7(1), 7-8.

Montero, I. \& León, O. G. (2007). A guide for naming research studies in Psychology. International Journal of Clinical and Health Psychology, 7, 847-862.

Muñiz, J. \& Fonseca-Pedrero, E. (2008). Construcción de instrumentos de medida para la evaluación universitaria. Revista de Investigación en Educación, $5,13-25$.

Musi-Lechuga, B., Olivas-Ávila, J. \& Buela-Casal, G. (2009). Producción científica de los programas de doctorado en Psicología Clínica y de la Salud de España. International Journal of Clinical and Health Psychology, 9, 161-173.

Musi-Lechuga, B., Olivas-Ávila, J., Portillo-Reyes, V. \& Villalobos-Galvis, F. (2005). Producción de los profesores funcionarios de Psicología en España en artículos de revistas con factor de impacto de la Web of Science. Psicothema, 17, 539-548

Navarrete-Cortés, J., Quevedo-Blasco, R., ChaichioMoreno, J. A. Ríos, C. \& Buela-Casal, G. (2009). Análisis cuantitativo por países de la productividad en psicología de las revistas en la Web of Science. Revista Mexicana de Psicología, 26, 131-143.

Osca-Lluch, J., Civera, C., Tortosa, F., Quiñones, E., Peñaranda, M., \& López, J. J. (2005). Difusión de las revistas españolas de Psicología en bases de datos nacionales e internacionales. Anales de Documentación, 8, 165-186.

Osuna Carrillo de Albornoz, E. (2009). Calidad y financiación de la universidad. Revista de Investigación en Educación, 6, 133-141.

Pagani, R., Vadillo, O., Buela-Casal, G., Sierra, J. C., Bermúdez, M. P., Gutiérrez-Martínez, O., Agudelo, D., Bretón, J. \& Teva, I. (2006). Estudio internacional sobre criterios e indicadores de calidad de las universidades. Madrid: ACAP.

Ramos-Álvarez, M., Moreno-Fernández, M. M., ValdésConroy, B. \& Catena, A. (2008). Criteria of the peer review process for publication of experimental and quasi-experimental research in Psychology: A 
guide for creating research papers. International Journal of Clinical and Health Psychology, 8, 751-764.

Rodríguez Marín, J., Mira, J. J., Galán, M., González, L., Gómez Gras, J. M., Valcárcel, M. \& Díez de Castro, E. (2009). La difusión de resultados en el ámbito universitario público. El papel de ciudadanos, gestores y financiadores. Revista de Investigación en Educación, 6, 153-159.

Ruiz Carrillo, E. \& Estrével Rivera, L. B. (2008). La ideología y la transformación del sujeto. Universitas Psychologica, 7(1), 33-41.

Ruiz-Pérez, R., Delgado, E. \& Jiménez-Contreras, E. (2006). Criterios del Institute for Scientific Information para la selección de revistas científicas. Su aplicación a las revistas españolas: metodología e indicadores. International Journal of Clinical and Health Psychology, 6, 401-424.

Sierra, J. C., Buela-Casal, G., Bermúdez, M. P. \& Santos, P. (2008). Análisis transnacional del sistema de evaluación y selección del profesorado universitario. Interciencia, 33, 251-257.

Sierra, J. C., Buela-Casal, G., Bermúdez, M. P. \& Santos, P. (2009a). Diferencias por sexo en los criterios y estándares de productividad científica y docente en profesores funcionarios en España. Psicothema, 21, 124-132.

Sierra, J. C., Buela-Casal, G., Bermúdez, M. P. \& Santos, P. (2009b). Importancia de los criterios e indicadores de evaluación y acreditación del profesorado universitario en los distintos campos de conocimiento de la UNESCO. Revista de Universidad y Sociedad del Conocimiento (RUSC), 6(2), 49-59.

Teodoro, M. L. M., Cerqueira-Santos, E., Araujo de Morais, N. \& Koller, S. H. (2008). Protective factors related to smoking among Brazilian youth. Universitas Psychologica, 7(1), 139-147.

Valenzuela, J. (2009). Características psicométricas de una escala para caracterizar el sentido del Aprendizaje Escolar. Universitas Psychologica, 8(1), 49-60.

Viñolas, B., Aguado, A., Josa, A., Villegas, N. \& Fernández Prada, M. A. (2009). Aplicación del análisis de valor para una evaluación integral y objetiva del profesorado universitario. Revista de Universidad y Sociedad del Conocimiento (RUSC), 6(2), 22-37.

Zárraga, A., Balluerka, N. \& Ferreira, E. (2009). Modelo de evaluación integral de la actividad docente, investigadora y gestora de los departamentos de la Universidad del País Vasco/Euskal Herriko Unibersitatea. Revista de Universidad y Sociedad del Conocimiento (RUSC), 6(2), 69-79.

Zych, I. \& Buela-Casal, G. (2007). Índice de Internacionalidad de las revistas iberoamericanas de psicología incluidas en la Web of Science. Revista Méxicana de Psicología, 24, 15-22.

Zych, I. \& Buela-Casal, G. (2009). The Internationality Index: Application to Revista Latinoamericana de Psicología. Revista Latinoamericana de Psicología, 41, 401-412. 